\title{
Some Aspects of the Metaphysics of Chemistry and the Nature of the Elements
}

\author{
Eric R. Scerri
}

\begin{abstract}
There is now a considerable body of published work on the epistemology of modern chemistry, especially with regard to the nature of quantum chemistry. In addition, the question of the metaphysical underpinnings of chemistry has received a good deal of attention. The present article concentrates on metaphysical considerations including the question of whether elements and groups of elements are natural kinds. It is also argued that an appeal to the metaphysical nature of elements can help clarify the re-emerging controversies among chemists regarding the placement of the elements hydrogen and helium in the periodic system and the question of whether there exists a best form of the periodic table.
\end{abstract}

Keywords: Metaphysics, element, periodic table, reference theory, Kripke E Putnam.

\section{Introduction}

A good deal of work carried out up to this point has been of an epistemological nature, such as the examination of the reduction of chemistry to quantum mechanics by a number of authors (e.g. Ramsey 1997, Needham 2000, Woody 2000, Scerri 2001). However, given the recent growth in the study of the philosophy of chemistry, it seems appropriate to consider also the metaphysical aspects of the field.

In the philosophy of physics, metaphysical considerations have been examined for some time and they continue to be the focus of much attention. For example Cao has devoted a great deal of effort in arguing that a metaphysical understanding of quantum field theory is essential in trying to comprehend the nature of quantum mechanics. While Cao argues for a physical ontology consisting of entities, others equally interested in the metaphysical foundations of physics argue that the ontology lies not in particles or entities but in the mathematical structure of quantum field theory (Cao 2003, French \& Ladyman 2003). In the philosophy of biology one of the topics of interest

HYLE - International Journal for Philosophy of Chemistry, Vol. 11 (2005), No. 2, 127-145. Copyright (0) 2005 by HYLE and Eric R. Scerri. 
has been the question of whether biological species are natural kinds or not (Ghiselin 1974, Hull 1977, LaPorte 2004). Several authors have appealed to the metaphysical foundations of biology in order to clarify such questions (Hull 1977).

In chemistry the prospects for a metaphysical project might appear to be gloomy at first sight. Whereas theoretical physics has been increasingly forced to embrace an abstract ontology that focuses attention on the vacuum fluctuations, a similar move has not occurred in chemistry. Chemistry generally speaking stops at non-relativistic or relativistic quantum mechanics and does not generally require the use of a full-blown quantum field approach and an appeal to the quantum mechanical vacuum. ${ }^{1}$

In addition, the appeal to metaphysical considerations appears to be undermined because many commentators have falsely claimed that chemistry has become a reduced science or a service science to physics and biology (Knight 1992, pp. 157-170; Bensaude-Vincent \& Stengers 1996, pp. 207-252). Indeed chemistry is regarded as an increasingly utilitarian science by many chemists themselves. The commonly held view that chemistry reduces to quantum mechanics would seem to obviate a need for a specifically chemical ontology or metaphysics. But now that these reductionist claims have been increasingly challenged in the philosophy of science, this would seem to clear the way for more work on the metaphysics of chemistry.

Various philosophers of chemistry have already examined metaphysical aspects of chemistry and in particular the question of the elements as natural kinds (Schummer 1996, Bensaude-Vincent 1998, van Brakel 2000, Cahn 2002, Harré 2005, Lombardi \& LaBarca 2005).

The present article will appeal to two interrelated metaphysical views concerning the chemical elements. The first such view is what may be called metaphysical in the naïve, or literal, sense of the elements as being beyond observation. The second metaphysical view considers the elements as the fundamental entities or natural kinds of chemistry. More work has been carried out regarding the latter, including the question of whether the elements actually represent natural kinds. For example, the Kripke-Putnam causal theory of reference has led to a good deal of discussion of precisely how elements should be referred to and the question of their essential properties. There are some interesting connections between these two metaphysical aspects of the elements, that will be indicated as the article proceeds. 


\section{First sense of metaphysics: Elements as unobserv- able substances}

A long-standing metaphysical view about the nature of elements has regarded them as basic substances or as bearers of properties. This view has never been completely abandoned in chemistry and I will argue that it can be brought up to date to serve our present needs. The chemical elements, in the unobservable sense, were first invoked by some Greek philosophers and continued to play a role up to the beginning of $20^{\text {th }}$ century chemistry, although in a modified sense.

The manner in which philosophically inclined chemists have regarded elements is concerned with the ancient question of how the elements survive, if at all, when they form compounds. This conundrum has been classically resolved, since the time of the ancient Greek philosophers, by appealing to the dual sense of the term 'element'. An element can be regarded as a 'simple substance' that can be isolated and that can take several different structural forms, such as diamond or graphite, in the case of carbon. In addition, an element can also be regarded, more fundamentally, as a 'basic substance', which is the bearer of properties while at the same time being devoid of properties. ${ }^{2}$ This metaphysical view, in the sense of elements as unobservable bearers of properties was partly abandoned as a result of the chemical revolution. Lavoisier found it more useful to concentrate on elements as observable simple substances that can be isolated. As many authors have argued, Lavoisier did not succeed in completely banishing the notion of elements as unobservable bearers of properties, but this question will not be pursued any further here (Siegfried 2002).

It appears that the notion of elements as basic substances made its comeback in the writing of Mendeleev who insisted that his periodic classification was primarily concerned with this sense of the term 'element' and not as observable simple substances. However, Mendeleev did not merely return to the ancient view whereby the elements as basic substances were completely devoid of properties or characteristics. For Mendeleev a basic substance possessed at least one attribute, namely its atomic weight which served to distinguish it from other elements and which was used to order the elements in a unique sequence.

The distinction between elements as basic substances and as simple substances was held to be of crucial importance by Mendeleev. There are many passages in his classic textbook, The Principles of Chemistry, in which he goes to great lengths to explain it.

It is useful in this sense to make a clear distinction between the conception of an element as a separate homogeneous substance, and as a material but invisible part of a compound. Mercury oxide does not contain two simple bodies, a gas 
and a metal, but two elements, mercury and oxygen, which, when free, are a gas and a metal. Neither mercury as a metal nor oxygen as a gas is contained in mercury oxide; it only contains the substance of the elements, just as steam only contains the substance of ice, but not ice itself, or as corn contains the substance of the seed but not the seed itself. [Mendeleev 1891, p. 23]

In the $20^{\text {th }}$ century Paneth, one of the founders of radiochemistry, similarly upheld the view that it is only the element in the second sense, as a basic substance, that survives when sodium and chlorine combine to create sodium chloride. On the other hand, sodium and chlorine, as simple substances, consist of a gray metal and a green gas respectively, properties that do not survive when these elements combine together as simple substances. However, drawing on the recent discoveries of physics and especially the work of Moseley, Paneth changed the characteristic of an element as a basic substance from its atomic weight to its atomic number (Paneth 1962).

In addition, Paneth's philosophical message was to suggest that chemists typically adopt an 'intermediate position' between what he termed naïve realism and a metaphysical view. At other times, Paneth described this contrast as being one between naive realism and the reductive view provided by physics. Such distinctions may sound rather unusual to modern philosophers and, of course, Paneth was not a professional philosopher, thus possibly accounting for his choice of terminology. However, what Paneth had to say on the nature of chemistry and the elements, coupled with the fact that he was responsible for the introduction of the current definition of a chemical element, obliges us to examine his philosophical views more closely. One might consider that Paneth's terminology is somewhat unfortunate since he was not contrasting realism in general with metaphysics as these positions are generally understood, nor was he contrasting realism with reductionism as generally understood. Instead, Paneth was contrasting a macroscopic view of chemical phenomena (naïve realism) with a microscopic description (reduction to physics). But it must also be appreciated that Paneth's view was centered on the contrast between elements as simple substances (naïve realism) and elements as basic substances (metaphysical in the sense of being beyond observation). For Paneth reduction via physics represented a process leading to less and less observability of the kind that is more familiar to the chemist. ${ }^{3}$

Paneth claimed that the chemist could afford to take a naïvely realistic attitude in the case of most observed properties. For example, the ore of mercury, called cinnabar, can be regarded as just being red even though the chemist knows that the red color can be further reduced to a specific range of frequencies of reflected light. But Paneth also claimed that such a form of naïve realism, as accepting elemental properties at face value, breaks down when the chemist is confronted with the question discussed earlier regarding the persistence of elements in compounds. In such a case, the chemist is 
forced to abandon the naive realistic view of elements as simple substances, and must maintain what he termed a metaphysical view ${ }^{4}$, in the sense of elements as unobservable basic substances. Paneth suggested that chemists generally operate at an intermediate position, which is neither fully naïvely realistic nor metaphysical, in his terminology. I suggest that, in spite of such terminological pitfalls, Paneth's view is philosophically consistent. ${ }^{5}$

It is proposed that Paneth's view of the elements might serve to illuminate a number of issues in contemporary philosophy of science as well as in chemistry. But before doing so it is important to summarize the changing view of elements as basic substance from that of the ancient Greeks through Mendeleev's and Paneth's views. For some Greeks philosophers the elements as basic substances were completely unobservable. For Mendeleev elements as basic substances remained "invisible" to cite his own word, but were characterized by one main property, namely atomic weight. It would appear that the elements as basic substances had lost their fully metaphysical characteristic of being completely unobservable, and devoid of properties, since they now possessed one important attribute. Similarly, Paneth's understanding of elements as basic substances did not imply complete unobservability and was not a thoroughgoing metaphysical view in the literal sense mentioned earlier. For Paneth elements as basic substances possessed one important attribute, namely atomic number. ${ }^{6}$

\section{Second sense of metaphysics: Elements as the basic entities of chemistry or its natural kinds}

The beginning of this section may be familiar territory to analytical philosophers but perhaps not so to a chemical audience. A brief historical sketch of the developments in this area will therefore be given.

The motivation for the causal theory of reference and its criteria for referring to natural kinds has been a central question of how language relates to the world. Traditionally, expressions such as proper names refer to or designate an object because the name was associated with some descriptive content about the object in question. Analogously, general terms or nouns such as 'tiger', 'acorn', or 'element' were considered to refer to objects by virtue of their 'sense' where the sense provides the description of the object (Frege 1892).

This conception of the relationship between language and reality came under criticism in the 1960s and 1970s from a number of philosophers (Donnellan 1966, Kripke 1972, Putnam 1975). The gist of this criticism was that a description associated with a name or term like 'tiger' did not provide necessary and sufficient conditions for its application to the object in question. 
For example, a speaker might be referring to a tiger although the creature in question did not have the familiar stripes, in the case of an albino tiger. Conversely, an anomalously colored creature might display the striped appearance of a tiger while belonging to an altogether different species.

Or one might consider an example from Kripke involving the proper name 'Aristotle'. According to the traditional theory of names, at least some of the descriptive concepts usually believed of Aristotle are regarded as being necessarily true of the famous philosopher. However, as Kripke points out, it would be odd to regard it as necessarily true that Aristotle was a teacher of Alexander, or the pupil of Plato, or that he was even a philosopher. Aristotle might not have actually played any of these roles had the course of history been different, claims Kripke. It was not essential for Aristotle that he should have engaged in any of these activities. In Kripke's terminology, the name 'Aristotle' refers rigidly to that man independently of any description associated with the name Aristotle. Similarly, Kripke believes that nouns like proper names are rigid designators. If 'gold' is such a rigid designator, it refers to the same stuff, independently of its superficial (descriptive) appearance. For Kripke 'gold' rigidly designates the element with atomic number 79.' It is not the description of gold in terms of being shiny or malleable etc. that determines whether some substance is gold but only its atomic number. Iron pyrite, as Kripke points out, is not gold because it does not possess an atomic number of 79 , regardless of whether its appearance might resemble that of gold.

Intuitions of this kind led some philosophers to require that facts about the world should be regarded as matters of objective causal relations in the world and not ideas that people have in their heads. In this context, the Kripke-Putnam causal theory of reference was born. It was now claimed that nouns that are meant to designate natural kind terms do not have their extension determined by descriptive concepts but by a causal chain beginning with the original baptism or naming of a sample object. In addition, the reference of the term is provided by whatever is dictated by the latest scientific research, hence the use of atomic number in the case of any particular element. This last feature led Kripke and Putnam to adopt Paneth's definition of elements in terms of atomic number, since this remains as the most up-to-date scientific definition of an element.

\section{Convergence of views?}

If we now consider how an element is specified in chemistry and in the theory of causal reference, we reach an interesting situation. In chemistry, as 
emphasized above, the understanding of the term 'element' was provided by Mendeleev, and later modified by Paneth, eventually leading to the definition of an element adopted by the International Union of Pure and Applied Chemistry. Elements are regarded as basic substances that are devoid of properties apart from having an atomic number which in any case is not macroscopically observable. Similarly, and for quite other reasons, Kripke and Putnam, approaching the issue with a different set of concerns, arrived at the conclusion that names of elements like 'gold' did not identify such samples by virtue of their descriptive content or 'properties'. They claimed that names connect with the world through one single criterion provided by modern chemistry, namely atomic number accompanied by a causal chain from the initial act of baptism.

The fact that the criterion for elementhood turned out to be the same, namely atomic number, is not so remarkable since the philosophers made a direct appeal to the scientific understanding of an element and therefore inherited atomic number straightforwardly. What is perhaps worth dwelling upon is the fact that both the chemical and philosophical understanding of an element involve a denial of its observable or descriptive attributes. In Paneth's chemical account an element, regarded as a basic substance, does not possess observable properties. ${ }^{8}$ In the case of Kripke and Putnam the descriptive properties are commonly believed to exist but are ignored when referring to the element. I suggest that that there is convergence between Paneth and the philosophers Kripke and Putnam, and that both parties are dealing with the elements as basic substances and that important aspect of the causal theory of reference amounts to a denial of descriptive aspects. As far as I am aware this feature has not been previously noticed.

I suggest that the two apparently different metaphysical senses of the term 'element', namely an element as an unobservable basic substance and an element as a natural kind, may actually converge in the final analysis.

\section{Putting elements as basic substances to work}

In the 1920s Paneth drew on the metaphysical essence of elements as basic substances in order to save the periodic system from a major crisis. Over a short period of time many new isotopes of the elements had been discovered, such that the number of 'atoms' or most fundamental units suddenly seemed to have multiplied. The question was whether the periodic system should continue to accommodate the traditionally regarded atoms of each element or whether it should be restructured to accommodate the more elementary isotopes that might now be taken to constitute the true 'atoms'. Paneth's 
response was that the periodic system should continue as it had done before, in that it should accommodate the traditional chemical atoms and not the individual isotopes of the elements.9 The reason why Paneth regarded isotopes as simple substances is that they were characterized by their atomic weights and, as it will be recalled, basic substances were characterized by atomic number alone in Paneth's scheme. ${ }^{10}$

Moreover, Paneth along with Hevesy provided experimental evidence in support of this choice for chemists. ${ }^{11}$ They showed that the chemical properties of isotopes of the same element were for all intents and purposes identical. ${ }^{12}$ As a result the chemist could regard the isotopes of any element as being the same simple substance even though such atoms might occur in different isotopic forms.

It is worth noting that in the case of this isotope controversy, Paneth's recommendation for the retention of the chemist's periodic table depended on the notion of elements as basic substances and not as simple substances. If chemists had focused on simple substances, they would have been forced to recognize the new 'elements' in the form of isotopes that were being discovered in rapid succession. By choosing to ignore these 'elements' in favor of the elements as basic substances, chemists could continue to uphold that the fundamental units of chemistry, or its natural kinds, remained as those entities that occupied a single place in the periodic system. This represents a parallel case to that of the persistence of the elements in compounds, a situation in which chemists must likewise abandon naïve realism in favor of what $\mathrm{Pa}-$ neth called the metaphysical view of elements in order to rationalize the situation..$^{13}$

\section{Groups of elements as natural kinds?}

Having argued, with Kripke and Putnam, that elements as defined by their atomic numbers represent natural kinds in chemistry, the question arises as to whether groups of elements appearing in the periodic table might also represent natural kinds. Could it be that there is some objective feature that connects all the elements that share membership to a particular group in the periodic system?

It would seem that the criterion for membership to a group is by no means as clear-cut as that which distinguishes one element from another. In the case of groups of the periodic table, it is the electronic configuration of gas phase atoms that seems to provide the criterion, although in neither a necessary nor a sufficient manner as has been argued in previous publications (Scerri 2004). However, one may also argue that the placement of the ele- 
ments into groups is not a matter of convention. If periodic relationships are indeed objective properties, as will be argued here, it would seem to suggest that there is one ideal periodic classification, regardless of whether or not this may have been discovered. This in turn would have a bearing on some recent questions regarding the placement of some elements within the periodic system. And if electronic configurations do not perfectly capture the fact that groups are natural kinds, this may merely indicate the limitations of the concept of electronic configurations. ${ }^{14}$

\section{The placement of $\mathrm{H}$ and $\mathrm{He}$ in the periodic system}

There has been considerable debate within chemistry in recent years as to the placement of the elements hydrogen and helium within the periodic system (Cronyn 2003). For example, hydrogen is similar to the alkali metals in its ability to form single positive ions. However, hydrogen can also form single negative ions, thus suggesting that the element might be placed among the halogens that display this type of ion formation.

In a recent article, Peter Atkins and Herb Kaesz have proposed a modification to the periodic table concerning the placement of the element hydrogen (Atkins \& Kaesz 2003). Contrary to its usual placement at the top of the alkali metals, and its occasional placement among the halogens, Atkins and Kaesz choose to position hydrogen on its own and floating above the table. In doing so these authors appear to overlook the further possibility of hydrogen's membership of the group that is usually headed by carbon, as has recently been argued in detail (Cronyn 2003).

But rather than considering the relative virtues of these three possible placements, let us consider the argument for the removal of hydrogen from the main body of the table a little more closely and from the perspective of the elements as basic substances. The widely held belief, among chemists, is that the periodic system is a classification of the elements as simple substances that can be isolated and whose properties can be examined experimentally. However, as I have emphasized, there is a long-standing metaphysical tradition of also regarding the elements as unobservable basic substances. ${ }^{15}$

Our current inability to place hydrogen in the periodic table in an unambiguous manner should not lead us to exclude it from the periodic law altogether, as Atkins and Kaesz seem to imply in removing hydrogen from the main body of the table. Hydrogen is as subject to the periodic law as all the other elements. I maintain that there is a 'fact of the matter' as to the optimum placement of hydrogen in the main body of the table and that this is 
not a matter of utility or convention that can be legislated as these and other authors have argued. That is to say groups of elements should be regarded, as should elements themselves, as natural kinds as suggested above.

Helium is traditionally regarded as a noble gas in view of its extreme inertness and is subsequently placed among the other inert gases in group VIII of the periodic system. However, in terms of its electronic configuration helium has just two outer electrons and might therefore be placed among the alkaline earth metals such as magnesium and calcium. Many periodic tables appearing in physics books do just that, as do many spectroscopic periodic systems (White 1934).

Surprising as it may seem, some chemists have even proposed chemical evidence for placing helium in this manner. Such arguments are based on the first-element rule which, in its simplest form, states that the first element in any group of the periodic system tends to show several anomalies when compared with successive members of its group. For example, in the p block, all the first-member elements show a reluctance to expand their octets of outershell electrons, while subsequent group members do so quite readily. ${ }^{16}$

In addition, there is a more sophisticated version of this first-member rule that also specifies the extent to which the first elements in the various blocks of the Periodic Table display anomalies. For example, Jensen writes,

It is also sometimes argued that $\mathrm{H}$ is placed above the table because it is totally unique in its properties. While it is true that $\mathrm{H}$ is unique relative to other IA elements, this is really a reflection of the systematic variation in the periodic table which shows that the elements in the later row of any new electronic block tend to show abnormalities relative to the elements in later rows of the same block, and that the degree of divergence decreases in the order s-block $>>$ p-block $>$ d-block $>$ f-block. [Jensen 1986].

Another chemist, Bent, has taken up the sophisticated first-element rule, sometimes claiming it to be the same as Jensen's. According to Bent, "A group's first member is the group's most distinctive member, the more so the narrowest the group's block" ${ }^{17}$ Bent considers the sophisticated first-element rule as an argument for the placement of helium in the s block. He believes that it is only by placing hydrogen and helium in the alkali metals and alkaline earths, respectively, that they fall into narrowest block in the Periodic Table. Bent believes that one would fail to see any manifestation of the sophisticated first-element rule, if one were to leave helium in the p block, as is done traditionally. However, the use of the first-member rule has also led to much disagreement among chemists. For example, Jensen, who was cited earlier, regards the rule as an argument against the placement of helium among the alkaline earths (Jensen 1986).

Rather than relying on specific properties of the elements as simple substances, it is suggested that we should concentrate on elements as basic sub- 
stances. One should seek some form of underlying regularity in order to settle the question of the placement of any element. Such a possibility is discussed in the next section, along with the question of the best possible form for the periodic system.

\section{Is there a best form for the Periodic Table?}

The periods in the currently most popular representation of the periodic system, the so-called medium-long form, are arranged so that each one begins with a new value of $n$, or the first quantum number (Figure 1). This value denotes the main shell of the most energetic electron in each case, in terms of the aufbau principle, that is used to 'build-up' the configuration of any particular atom. In macroscopic chemical terms, the medium-long form table places the reactive metals, such as the alkalis and alkaline earths, on the left side of the Periodic Table and the reactive non-metals on the right side.

The conventional medium-long form displays the periods as though the main-shell number were the dominant criterion for the build up of successive periods. However, as is well known, this form of display leads to a somewhat confusing layout whereby in several cases a main shell begins to fill, followed by an interruption due to a transition metal series in which a penultimate shell is filled. Only after such interruptions, which are more pronounced in the case of periods that also include inner transition elements, does the filling of the main shell resume.

Many authors have suggested that a more satisfactory representation can be obtained by basing the start of periods on $n+l$ instead of $n .{ }^{18}$ This implies that the $s$ block must be shifted to the right of the p block elements, which leads more specifically to at least two particular Periodic Tables. The first one is the so-called left-step periodic table (Figure 2). The second is a modified form of the pyramidal periodic system that likewise places the $s$ block elements on the right hand edge of a pyramid (Figure 3). Both of these tables display two short periods of two elements thus satisfying the desire for regularity that many authors, including some group theorists, believe might lie at the heart of the periodic system (Neubert 1970).

Both of these alternative representations of the periodic system shown in Figures 2 and 3 display the elements in a continuous manner with no break between any sets of elements, contrary to what is encountered in the currently accepted medium-long form. But these tables also contain a feature that causes many chemists some concern, in that the element helium is firmly located among the alkaline earth elements. 


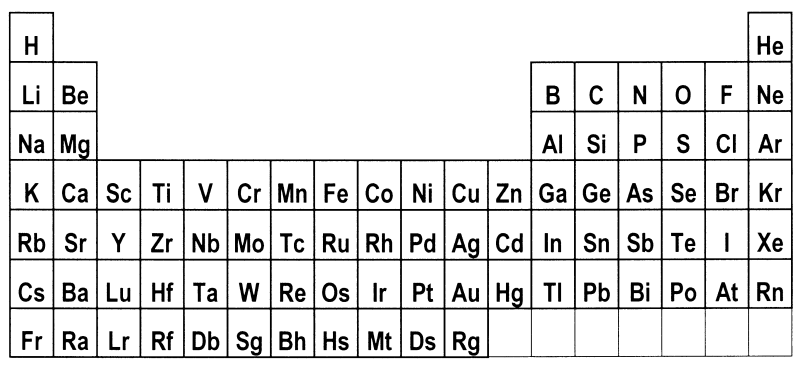

$$
\begin{array}{|l|l|l|l|l|l|l|l|l|l|l|l|l|l|}
\hline \text { La } & \mathrm{Ce} & \mathrm{Pr} & \mathrm{Nd} & \mathrm{Pm} & \mathrm{Sm} & \mathrm{Eu} & \mathrm{Gd} & \mathrm{Tb} & \mathrm{Dy} & \mathrm{Ho} & \mathrm{Er} & \mathrm{Tm} & \mathrm{Yb} \\
\hline \mathrm{Ac} & \mathrm{Th} & \mathrm{Pa} & \mathrm{U} & \mathrm{Np} & \mathrm{Pu} & \mathrm{Am} & \mathrm{Cm} & \mathrm{Bk} & \mathrm{Cf} & \mathrm{Es} & \mathrm{Fm} & \mathrm{Md} & \mathrm{No} \\
\hline
\end{array}
$$

Figure 1: Medium-long form of the Periodic Table.

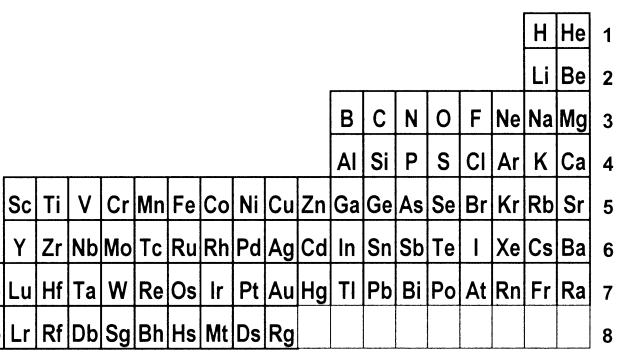

Figure 2: The left-step or Janet Periodic Table; numbers on the right represent values of $n+l$.

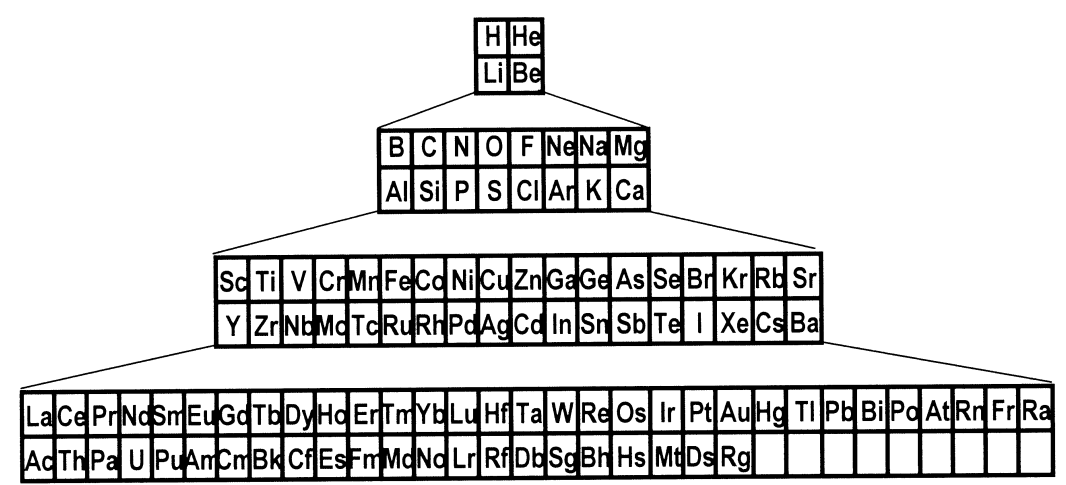

Figure 3: A modified pyramidal version of the Periodic Table. 
However, as I have argued in the previous section, such worries are alleviated once one acknowledges that the periodic system is primarily intended to classify the elements as basic substances and not simple substances.

Moreover, I claim that there is a fact of the matter concerning the best form of the periodic system in the sense that all elements belong in a particular group. Periodicity applies to all the elements, or in philosophical terms, to groups of elements which represent natural kinds. I do not agree with some chemists who consider the representation of the periodic system as a matter of convention as exemplified by the quotations below. In the preface of his well-known book that compiles the various forms of the periodic system produced up to the year 1970, Edward Mazurs writes (1974, p. xi),

The third section, the main part of the book, is based on a survey and analysis of the approximately seven hundred periodic tables published during the past one hundred years. The number and variety of these charts represent the ability of the human mind to give disparate forms to the same body of matter.

Similarly, in a recent article on a new presentation of the periodic system, the author writes (Stewart 2004, p. 156),

Of the making of Periodic Tables there is no end. No version can ever be definitive because there are various incompatible objectives. Some authors provide a schematic version that is readable and easily reproduced, while others exploit devices such as the third dimension to express complexity. Some aim at simplicity or grace while others want to convey detailed information on such things as relative atomic mass, valency, electronic structure, melting and boiling points, electronegativity, radioactivity, metallic or non-metallic nature, geological affinities and so on.

The chemist Henry Bent (2004, p. 7) writes,

One might wonder - which periodic table is best? As impossible as unnecessary to say? Best for what purpose(s)? Location of the problem elements? The left-Step Table. Discussion of horizontal trends in metal/non-metal character? The Left-step Table. Discussion of the most familiar elements, with beginning students? The Conventional [medium-long form] table [...] Graphic display of secondary kinships? Neither table. Better is Mendeleev's "Short Form".

Although one can partly agree with the view that different representations can help to convey different forms of information, I believe that one may still maintain that one particular representation reflects chemical periodicity, regarded as an objective fact, in the best possible manner. I am thus suggesting a realist view of the periodic law that requires believing that groups of elements, as well as elements themselves, are natural kinds. 


\section{Conclusions}

The metaphysical notion (unobservable sense) of the elements as basic substances has been historically important in the case of Mendeleev's establishment of the periodic system and Paneth's resolution of the fate of the periodic system in light of the discovery of isotopes. A seemingly different metaphysical notion of elements in which they are regarded as natural kinds has been developed in philosophy in response to concerns about the manner in which names and scientific terms refer to objects in the world. It has been suggested in the present article that the two approaches may converge, thus implying that elements as basic substances are being invoked in the domains of chemistry as well as philosophy when discussing the elements.

It has also been suggested that the notion of elements as basic substances can cast some light on the question of the best possible representation of the periodic system. As in the case of the distinction between elements as basic substances and as simple substances, the primary aim should be to classify elements as basic substances, while also recognizing aspects of the elements as simple substances. This optimal classification will not be obtained by behaving as naïve inductivists and by agonizing over the minutiae of the properties of hydrogen, helium, or other problematic elements, as Jensen seems to favor (Jensen 2003). It is suggested that it will be obtained by identifying the deepest and most general principles that govern the atoms of the elements, such as the $n+l$ rule, and by basing the representation of the elements on such principles. ${ }^{19}$

Just as philosophically inclined chemists, like Mendeleev and Paneth, have resolved some major conceptual issues by appeal to the metaphysical nature of the elements, so can the contemporary philosopher of chemistry. Although all comments have thus far been restricted to the nature of the chemical elements, the appeal to a metaphysical approach should be promising in other areas of philosophy of chemistry, given the centrality of the elements in this field.

The above analysis is not intended to imply that the Kripke-Putnam theory of reference is free of any criticism. For example, two prominent philosophers of chemistry have objected to it on the basis that it relies too heavily upon micro-reduction. For example, van Brakel espouses a radical antireductionism in which he favors the 'manifest image' over micro-reduction. His writings have included a thoroughgoing critique of Kripke's and Putnam's theory and a review of all other critiques that have been made in the context of chemical kinds (van Brakel 2000). Similarly, Needham takes an anti-reductionist approach and prefers to define substances through the Gibbs phase rule (Needham 2005). 
In addition some mainstream philosophers of science have pointed out the so-called qua-problem that has become to be viewed as increasingly problematic for the Kripke-Putnam account of reference (Devitt \& Sterelny 1978, Stanford \& Kitcher 2000). According to the Kripke-Putnam view, the scientific term 'gold', for example, involves ostentation of samples of the substance gold that possess an atomic number of 79 . However, it is not clear qua member of which kind such a sample of gold is being ostended. It could be as a metal, as a solid, as a shiny substance, or any number of possibilities other than possessing atomic number 79. The response from some philosophers has been to suppose that the Kripke-Putnam view needs to admit some relaxation of its semantic externalism (Stanford \& Kitcher 2000).

More recently a philosopher of chemistry, Hendry, claims to have an approach which can counter the qua-problem but without any relaxation of Kripke and Putnam's externalism (Hendry 2005). Hendry believes that what matters when scientists are referring to elements, for example, is their intentions. Indeed, Hendry's main example consists of an analysis of the term 'element' as used by a number of famous chemists. Hendry claims that the sense of the term appears to change if one considers the writings of Boyle, Lavoisier, Mendeleev, and Paneth. But he also claims that if we consider what these chemists intended by the term in their work, rather than what they actually claimed they were doing, then we will discover that there has been a continuity of reference with regard to the term 'element'. As I see it, there are some serious problems with Hendry's account which will form the basis of a forthcoming article (Scerri, forthcoming). Nevertheless I agree with this author that there has been a continuity of reference but disagree with the manner in which Hendry has attempted to establish this notion.

In spite of all these apparent shortcomings with the Kripke-Putnam causal theory as they apply to the term 'element', it is not my present task to analyze these problems. The purpose of this article has been to draw parallels between two senses in which the elements may be regarded metaphysically. The purpose has been to also draw parallels between the Mendeleev-Paneth definition of an element as a basic substance on the one hand, and the Kripke-Putnam account of elements as natural kinds on the other hand, both of which approaches deny observable properties to the elements. Finally, the purpose has been to apply the concept of elements as basic substances, which is explicit in the chemical definition and perhaps implicit in the KripkePutnam theory of reference, to contemporary issues concerning the placement of certain elements in the periodic system. Of course the overall purpose has been to stimulate further discussion on these issues which lie at the foundations of chemistry. 


\section{Dedication}

This article is dedicated to Fernando Dufour, a leading pioneer of the periodic system, on the occasion of his $80^{\text {th }}$ birthday.

\section{Notes}

1 Exceptions to the lack of use of QFT in chemistry include the work on the origins of optical activity (Barron 1982).

2 This particular terminology is due to Paneth. Although this sense of the term 'element' may sound somewhat mysterious, it should be pointed out that it was essential to Mendeleev's ordering of the elements in the periodic system. For example, if he had restricted himself to the properties of simple substances, he might not have grouped together fluorine, chlorine, bromine, and iodine, which are respectively a yellow gas, a green gas, a brown liquid, and a violet-black solid. It is rather the compounds of these elements that show close similarities. Here the elements are present as basic substances.

3 This sense of naïve realism is sometimes explained in terms of 'Eddington's table'. As Eddington wrote, if we remain at the macroscopic level, a wooden table appears to be solid and impenetrable. But if we consider the view from the perspective of a reductive physics, we learn that the atoms that make up the table are mostly empty space, a view that appears difficult to reconcile with the solidity of the table.

4 Paneth also used the term 'transcendental elements' to denote the elements as basic substances, a term borrowed from the $19^{\text {th }}$ century German philosopher von Hartmann (Paneth 1962).

5 The position might not have been so coherent if Paneth had been speaking of completely unobservable elements, as did some ancient and medieval philosophers.

6 Robin Hendry has argued that Paneth is incorrect in his use of the word 'metaphysical' since Paneth is only referring to 'more abstract' elements in contrast to the more concrete simple substances (Hendry 2005).

7 Although Kripke allows that it is an empirical hypothesis that gold is the element with atomic number of 79 , he also claims that if this hypothesis is correct then 'gold' rigidly designates the element with this atomic number.

8 Apart from atomic number which is detectable, if not directly observable.

9 Another radiochemist, Kasimir Fajans, was Paneth's leading opponent in believing that the periodic system would not survive the discovery of isotopes.

10 A reviewer has suggested that if Paneth's opponent Fajans had been willing to accept the notion of elements as basic substances, he could equally well have regarded the isotopes as basic substances. Although this possibility would appear to be plausible on philosophical terms, it could not have been an option for Fajans because it would have implied a return to the notion of characterizing the elements in terms of atomic weight rather than atomic number. The use of atomic number, discovered by Moseley and championed in chemistry by Paneth, solves 
the problem of pair reversals. For example, the elements tellurium and iodine fall in chemically incorrect places on the periodic table if they are ordered according to atomic weight. The use of atomic number justifies their reversal as was carried out by the pioneers of the periodic system. From this point on, elements were identified by their atomic numbers and not their weights. The discovery of isotopes that differ in terms of weights does not alter the fact that elements as basic substances were henceforth to be characterized by atomic numbers. Isotopes are examples of simple substances since they represent instances of the elements that can actually be isolated, by contrast with the abstract atom of any particular element that occupies a space in the periodic table.

11 Paneth and Hevesy showed that the electrochemical potential from two cells made from different isotopes of the metal bismuth was the same as far as experimental techniques of the day could distinguish (Scerri 2000).

12 The fact that more recent research has revealed some differences even in the chemical properties of isotopes does not alter the central issue under discussion.

13 Paneth did not fully commit himself on the question of metaphysical elements. He merely stated that he is using the terminology developed by philosopher von Hartmann (Paneth 1962).

14 Electronic configurations are known to be approximations, unlike atomic number which can be given a clear realistic interpretation in terms of the number of protons in the nucleus of any atom (Scerri 2004).

15 Once again unobservable apart from their possessing an atomic number.

16 For example, oxygen, a first member element, forms $\mathrm{OF}_{2}$ with eight electrons in the outer shell of oxygen. However, sulfur the second member of the group readily forms $\mathrm{SF}_{6}$ in which twelve electrons occur in the outer shell of the sulfur atom (octet expansion).

17 Bent, in preparation; what Bent has actually written here is "the shorter the group's block". The word 'shorter' normally indicates the vertical dimension of a block which is not what the author intended. This error was later confirmed to me by the author via personal correspondence.

18 Janet 1929, Simmons 1948, Sanderson 1964, Katz 2001. The $l$ or second quantum number is related to the $n$ quantum number. For any given value of $n$, the value of $l$ can adopt any integral value from 0 up to and including the value $n-1$.

19 As in the use of atomic number, the use of the $n+l$ rule appeals to elements as basic substances and not as simple substances. This rule represents a generalization concerning all the elements, although it is violated in some instances, and is not concerned with any directly observable properties of the elements.

\section{References}

Atkins, P.W., Kaesz, H.: 2003, 'The Placement of Hydrogen in the Periodic Table', Chemistry International, 25, 14-14.

Barron, L.D.: 1982, Molecular Light Scattering and Optical Activity, Cambridge UP, Cambridge.

Bensaude-Vincent, B.: 1998, Éloge du Mixte, Hachette, Paris.

Bent, H.: (in preparation), Journal of Chemical Education. 
Cahn, R.M.: 2002, Philosophische und historische Aspekte des Periodensystems der chemischen Elemente, HYLE Publications, Karlsruhe [www.hyle.org/publications/books/cahn/index.html]

Cao, T.Y.: 2003, 'Structural Realism and the Interpretations of Quantum Field Theory', Synthese, 136, 3-24.

Cronyn, M.W.: 2003, 'The Proper Place for Hydrogen in the Periodic Table', Journal of Chemical Education, 80, 947-51.

Devitt, M. \& Sterelney, K.: 1987, Language and Reality, MIT Press, Cambridge, MA.

Donnellan, K.: 1966, 'Speaking of Nothing', The Philosophical Review, 83, 3-32.

Frege, G.: 1892, 'Über Sinn und Bedeutung', Zeitschrift für Philosophie und philosophische Kritik, 100, 25-50 [English: 'On Sense and Reference', trans. M. Black, in: P. Geach \& M. Black (eds.), Translations from the Philosophical Writings of Gottlob Frege, 3rd edn., Blackwell, Oxford, 1980].

French, S. \& Ladyman, J.: 2003, 'Remodelling Structural Realism: Quantum Physics and the Metaphysics of Structure', Synthese, 136, 31-56.

Ghiselin, M.: 1974, 'A Radical Solution to the Species Problem', Systematic Zoology, $23,536-44$

Harré, R.: 2005, 'Chemical Kinds and Essences Revisited', Foundations of Chemistry, 7, 7-30.

Hendry, R.F.: 2005, 'Lavoisier and Mendeleev on the Elements', Foundations of Chemistry, 7, 31-48.

Hull, D., 1977.: 'The Ontological Status of Species as Evolutionary Units', in R. Butts \& J. Hintikka (eds.), Foundational Problems in the Special Sciences, Reidel, Dordrecht, 1977, pp. 91-102.

Janet, C., 1929, 'The Helicoidal Classification of the Elements', Chemical Nerws, 138, 372-374, 388-393.

Jensen, W.B.: 1986, 'Classification, Symmetry and the Periodic Table', Computation and Mathematics with Applications, 12B, 487-510.

Jensen, W.B.: 2003, 'The Place of Zinc, Cadmium and Mercury in the Periodic Table', Journal of Chemical Education, 80, 952-961.

Katz, G.: 2001, 'The Periodic Table: An Eight Period Table for the 21st Century', The Chemical Educator, 6, 324-332.

Knight, D.: 1992, Ideas in Chemistry, Rutgers UP, New Brumswick, NJ.

Kripke, S.: 1972, 'Naming and Necessity', in: D. Davidson \& G. Harman (eds.), Semantics of Natural Language. Reidel, Dordrecht, pp. 253-355.

LaPorte, J.: 2004, Natural Kinds and Conceptual Change, Cambridge UP, New YorkCambridge.

Lombardi, O. \& LaBarca, M.: 2005, 'The Ontological Autonomy of the Chemical World', Foundations of Chemistry, 7, 125-148.

Mazurs, E.: 1974, Graphic Representation of the Periodic System During One Hundred Years, Alabama UP, Tuscaloosa, AL.

Mendeleev, D.I.: 1891, The Principles of Chemistry, Longmans, Green and Co., London [first English translation from the fifth Russian edition].

Needham, P.: 2000, 'Reduction and Abduction - A Response to Scerri', International Studies in the Philosophy of Science, 14, 317-323.

Needham, P.: 2005, 'Mixtures and Modality', Foundations of Chemistry, 7, 103-118.

Neubert, D.: 1970, 'Double Shell Structure of Periodic System of Elements', Zeitschrift fur Naturforschung, 25A, 210-217.

Paneth, F.A.: 1920, 'Die neueste Entwicklung der Lehre von den chemischen Elementen', Naturwissenschaften, 8, 839-842, 867. 
Paneth, F.A.: 1962, 'The Epistemological Status of the Concept of Element', British Journal for the Philosophy of Science, 13, 1-14, 144-160, trans. H.R. Post [reprinted in Foundations of Chemistry, 5 (2003), 113-145].

Putnam, H.: 1975, 'The Meaning of "Meaning”' in: Philosophical Papers, vol. 2, Cambridge UP, Cambridge.

Sanderson, R.T.: 1964, 'A Rational Periodic Table', Journal of Chemical Education, 41, 187-189.

Scerri, E.R.: 2000, 'Naive Realism, Reduction and the 'Intermediate Position', in: N. Bhushan \& S. Rosenfeld (eds.), Of Minds and Molecules, Oxford UP, New York, pp. 51-72.

Scerri, E.R.: 2001, 'The Recently Claimed Observation of Atomic Orbitals and Some Related Philosophical Issues', Philosophy of Science, 68 (Proceedings) S76-S88.

Scerri, E.R.: 2004, 'How Ab Initio is Ab Initio Quantum Chemistry?', Foundations of Chemistry, 6, 93-116.

Scerri, E.R.: (forthcoming), 'On the Continuity of Reference of the Elements. A Response to Hendry', Studies in History and Philosophy of Science.

Schummer, J: 1996, Realismus und Chemie. Philosophische Untersuchungen der Wissenschaft von den Stoffen, Königshausen \& Neumann, Würzburg.

Siegfried, R.: 2002, From Elements to Atoms: A History of Chemical Composition, American Philosophical Society, Philadelphia, PA.

Simmons, L.M.: 1948, 'A Display of Electronic Configuration by a Periodic Table', Journal of Chemical Education, 25, 658.

Stanford, P.K. \& Kitcher, P.: 2000, 'Refining the Causal Theory of Reference for Natural Kind Terms', Philosophical Studies, 97, 99-129.

Stewart, P.: 2005, 'A New Image of the Periodic Table', Education in Chemistry, 6, 156-158.

Van Brakel, J.: 2000, Philosophy of Chemistry, Leuven UP, Leuven.

White, H.E.: 1934, Introduction to Atomic Spectra, McGraw-Hill, New York.

Woody, A.: 2000, 'Putting Quantum Mechanics to Work in Chemistry: The Power of Diagrammatic Representation', Philosophy of Science, 67 (Proceedings), S612S627.

Eric R. Scerri:

Department of Chemistry \& Biochemistry, UCLA, Los Angeles, CA 90095-1569,USA; scerri@chem.ucla.edu 